\title{
Leukocytosis of Unknown Etiology in a Patient with a Spinal Cord Stimulator
}

\author{
Jie Zhu ${ }^{1,2,3 *}$, Jessica Arias Garau ${ }^{4}$ and Carlos E. Calvo ${ }^{5}$ \\ ${ }^{1}$ Mid Atlantic Spine \& Pain Physicians, Newark and Milford, DE and Elkton, Maryland, USA \\ ${ }^{2}$ Pain Medicine Fellowship Program, Temple University Hospital Philadelphia, USA \\ ${ }^{3}$ Temple University Medical School, Philadelphia, USA \\ ${ }^{4}$ Advanced Orthopedics and Sports Medicine Institute, Freehold. NJ. Faculty, Pain Medicine Fellowship Program, Temple University Hospital, Philadelphia, USA \\ ${ }^{5}$ Mid Atlantic Spine \& Pain Physicians, Newark and Milford, DE and Elkton, MD Fellow, Pain Medicine Fellowship Program, Temple University Hospital, Philadelphia, USA
}

Failed back surgery syndrome (FBSS) is very common in the United States. The prevalence of FBSS in the United States is not exactly known, however, of all patients undergoing lumbar surgery, an estimated 10$15 \%$ of them still suffer from the persistence of pain and disability following unsuccessful back surgery(s). [1]. FBSS impacted health care and has been a significant cause of disability in patients suffering from low back pain [1]. Despite the improvement in surgical techniques and technology, FBSS continues to prevail [2-4]. Numerous modalities have been used to treat FBSS, including injections, physical therapy and medications, but there is a subset of patients that persist with pain. These patients might potentially benefit from neuromodulation with a spinal cord stimulator (SCS) to alleviate their symptoms [5]. SCS is indicated for patients with radicular or neuropathic pain (nerve root injury, CRPS, arachnoiditis, etc). It is also beneficial for axial back pain in patients suffering from FBSS and other conditions such as peripheral vascular disease.

We present a case of a 42-year-old female who has undergone multiple lumbar surgeries for the treatment of FBSS. A spinal cord stimulator was implanted due to a gradual increase in chronic low back and radicular leg pain. This patient had a history of leukocytosis even prior to the initial lumbar surgery, which continued to increase after the first lumbar fusion and SCS implant. Following strong recommendations from an infectious disease specialist due to persistent leukocytosis of an unknown etiology, the SCS was removed. The patient did have significant relief of back and leg pain from the SCS implant for three years and seven months before it was removed and underwent another lumbar fusion soon after SCS removal.

\section{Case Report}

A 42-year-old African American female patient was seen in our clinic for more than ten years for chronic low back pain. She presents a past medical history of intractable low back and bilateral radicular leg pain, secondary to severe degenerative disc disease, spondylosis and lumbar radiculopathy. On physical examination, the patient had an antalgic gait and was ambulating with a cane due to persistent back and leg pain.

The patient presented a history of leukocytosis prior to her initial visit at our clinic and previous to the first lumbar surgery on 2003. The duration of the leukocytosis was unable to be determined due to lack of documentation. The patient's WBC count prior to the initial surgery was 13 per $\mathrm{nl}$, at this time she was not presenting any symptoms suggestive of infection.

On 10/23/2003 this patient underwent bilateral L4-L5 hemilaminotomies and foraminotomies, with L4-L5 microdiscectomy, which provided back and leg pain relief for about a year.

Gradually the low back and radicular leg pain worsened, requiring several steroid spinal injections as well as chronic opioid management. On 2/21/2007, an MRI of the lumbar spine without contrast showed degeneration of the L4-5 disc with moderate to large broad based right paracentral disc extrusion, extending slightly inferiorly, deforming the thecal sac, and compressing the right L5 nerve root. Degeneration of the L5-S1 disc with a small central disc protrusion was also noted. The patient obtained some relief with interventional procedures and opioid pain management temporarily, but the low back and leg pain gradually increased.

The patient underwent a second lumbar surgery on $4 / 23 / 2007$, which included a bilateral L4-5 microdiskectomy, right L4 laminotomy and medial facetectomy with bilateral foraminotomy, right L5 laminectomy and foraminotomy, without relief. During this time, The patient's WBC count fluctuated between 10.6 and 12.6.

The 2nd lumbar surgery did not render the patient with good relief of the low back or leg pain. Subsequently, the patient required a 3rd lumbar surgery on 11/25/2008, which included a bilateral L4L5 reoperative laminotomy, medial facetectomy of L4-5, far lateral foraminotomy and microscopic microdissection, L4-L5 instrumented fusion, open reduction for collapsed L4-L5 disc with pedicle screw instrumentation and cage placement. This surgery was also unsuccessful in providing relief of the low back and leg pain, even after receiving additional opioid treatment and spinal interventional procedures, intermittently.

Following surgery, the WBC count increased to 14.6, and continued to fluctuate with an increasing trend. Medical clearance was obtained, and then the patient underwent a successful SCS trial on $9 / 14 / 2009$, followed by implantation on $10 / 26 / 2009$. The patient then presented to the emergency room after several episodes of fever on 6/4/2010. At that time, the WBC count increased to 17.2. The patient had C-reactive protein elevated to 18.8 , however, the soft tissue ultrasound performed during the emergency room visit did not show fluid collection or masses at SCS leads and battery sites. The emergency room physician could not find any signs of infection on physical examination. The patient was discharged home with oral antibiotics for possible urinary tract infection due to symptoms of increased urinary frequency and burning sensation.

Following the SCS implant, the patient reported significant relief of the radicular pain and some of the back pain. After the emergency room visit, she was referred to an infectious disease (ID) specialist who ordered a whole body white cell scan. The study showed no abnormal findings suggestive of a spinal infection.

A hematology/oncology specialist also evaluated the patient and could not find evidence of infection, leukemia or any other causes that

*Corresponding author: Jie Zhu, Mid Atlantic Spine \& Pain Physicians, 139 East Chestnut Hill Road, Newark, DE, 19713, USA, Tel: (302)369-1700; Fax: (302)3691717; E-mail: zzzzjim@gmail.com

Received December 03, 2014; Accepted December 29, 2014; Published December 31, 2014

Citation: Zhu J, Garau JA, Calvo CE (2014) Leukocytosis of Unknown Etiology in a Patient with a Spinal Cord Stimulator. J Spine 4: 204. doi: 10.4172/2165-7939.1000204

Copyright: () 2014 Zhu J, et al. This is an open-access article distributed under the terms of the Creative Commons Attribution License, which permits unrestricted use, distribution, and reproduction in any medium, provided the original author and source are credited. 
could explain the findings, therefore observation was advised.

The patient's WBC count reached an apex of 22.8 on 12/22/2010. She continued to follow-up with the ID specialist, who could not provide any definite diagnosis for the leukocytosis. The WBC count then stabilized to levels around $15-17$ from $01 / 2011$ to $01 / 2013$. During this time, the patient continued to receive significant relief of the radicular pain from the SCS implant.

The infectious disease specialist recommended an explant of the SCS, even though the patient was not presenting any physical signs or symptoms of infection. These recommendations were given after the patient's leukocytosis increased to 16.8 on 1/30/2013. Lengthy discussions were carried out with the patient regarding the chronic leukocytosis of unknown etiology, as well as the ID recommendations. After all questions were addressed, the patient agreed to have the SCS removed. There were no any signs of infection found intraoperatively (Figure 1).

Medical clearance was obtained and the patient underwent the SCS explantation on $5 / 13 / 2014$. This procedure was executed after the ID specialist gave strong recommendations for explantation, even though the etiology of the leukocytosis at this moment was unclear. Following the removal of the device, the patient's low back pain and radicular leg pain worsened.

She underwent a second lumbar fusion on 7/18/2013 due to unbearable back and leg pain after SCS removal. The surgery included an exploration and revision of the L4-5 spinal fusion, removal of L4-L5 posterior instrumentation, bilateral L4-L5 reoperative laminotomies, medial facetectomies of L4-L5 and far lateral foraminotomies, bilateral S1 laminotomies, foraminotomies, bilateral L5-S1 microdiscectomy, L5-S1 fusion with instrumentation and cage spacer, and an L4-L5-S1 posterolateral bone graft arthrodesis. After the second lumbar fusion, the leukocytosis returned to 15.4, and has remained elevated since then.

After the surgery, the patient continued to follow up with the ID specialist who ordered a whole body Gallium-67 scan on 10/18/2013, showing evidence of a bilateral lung activity worrisome for infection or inflammation. The study was followed by a chest CT scan on $1 / 23 / 2014$, which demonstrated a benign thymoma, which will be surgically removed in the future. Currently, the patient continues to follow up with the ID specialist, as well as the oncologist, who recommended a bone marrow biopsy for further evaluation. Since then the WBC count has remained around 15 .

\section{Discussion}

Throughout the years, FBSS has been treated with multiple modalities such as opioids, physical therapy, and interventional techniques, which have resulted in moderate improvement, but has left many patients with residual symptoms and persistent intractable pain.

In the case we presented, a 42 year-old African American female underwent 3 lumbar surgeries and a SCS implant within a period of 10 years due to severe lumbosacral discogenic disease, and radiculopathy. This patient had a history of leukocytosis prior to the initial surgery, but after the first lumbar fusion, an increasing pattern of WBC count was observed. The leukocytosis continued to fluctuate until it reached a peak of 22.8, prompting the removal of SCS implant even though there was no direct evidence of infection at the SCS implant sites.

There are multiple causes that could be inciting this patient's leukocytosis. One possibility could be attributed to any surgical and or hardware complications from the SCS. A systematic review by Taylor et al. [6] reported adverse effects and complications associated to SCS including electrode or lead problems (27\%), generator problems $(6 \%)$, and extension cable problems (10\%). Turner et al. [7] reported that the most common surgical complication is infection (5\%), usually seen within one year after implantation. In a study by Engle et al. [8], the rate of infection correlated with the duration of the surgery. Other studies have shown that repeated spinal surgeries can result in a higher rate of surgical site infections [9].

In addition to an infectious process, an allergic or rejection reaction to the materials in the SCS could also be considered. Despite the technological progress achieved in the recent years, the implantable device functionality and durability can be compromised due to the host's response to the presence of a foreign body, as well as the tissue trauma caused during the surgery $[10,11]$. Hematologic causes such as myelodysplastic disorders, malignancies and leukemoid reactions cannot be excluded. Exposure to steroids may also promoteleukocytosis, but since this patient received sporadic spinal interventional procedures, the possibility is less likely. Finally, despite having the SCS explanted, the WBC count remained elevated at 15 and continued to fluctuate around this level (Figure 2 and Table 1). Therefore, the likeliness that the etiology of the leukocytosis is secondary to the SCS is remote. At present, this patient will be undergoing a bone marrow biopsy for further evaluation of any hematologic etiology.

A similar case of another 43-year-old African American female with a history of chronic low back, tailbone and radicular leg pain presented to our clinic with persistent leukocytosis fluctuating between 14.2 and 15.0 without any history of back surgery. This patient was scheduled for a SCS trial on two separate occasions, one year apart. Even though the patient was medically cleared to proceed with the SCS trial, the cases were cancelled by our surgical center staff who did not feel comfortable providing anesthesia for a patient with persistent leukocytosis. Both cases demonstrate patients with comparable age, gender, race, and physical complaints with a history of leukocytosis. One patient underwent SCS implant and explant, the other had an indication for SCS but the trial was cancelled.

\section{Conclusion}

In the case presented, it is dubious whether or not the etiology of the patient's leukocytosis was related to the presence of the SCS,

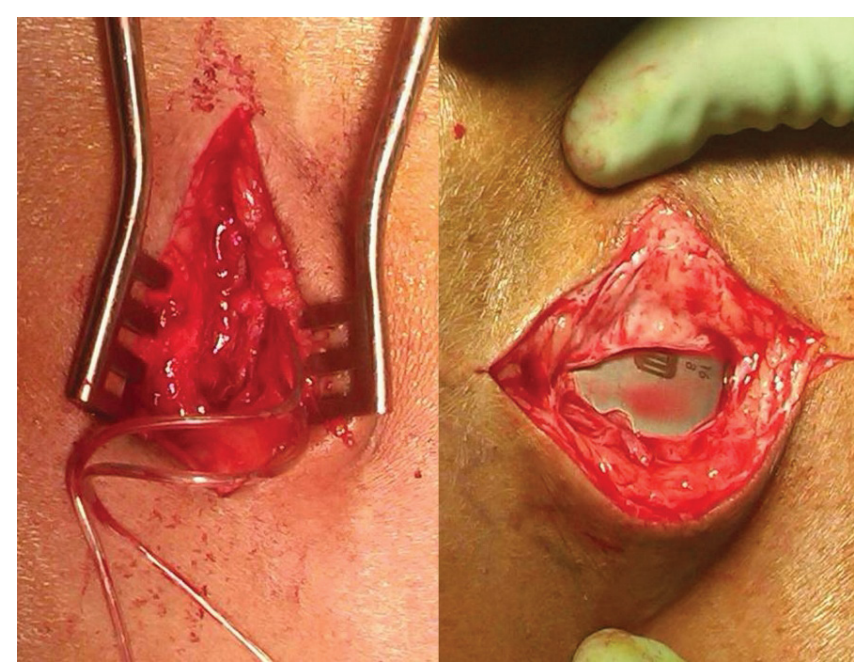

Figure 1: There is no gross evidence of infection at SCS implant sites found intraoperatively. 
Citation: Zhu J, Garau JA, Calvo CE (2014) Leukocytosis of Unknown Etiology in a Patient with a Spinal Cord Stimulator. J Spine 4: 204. doi:10.4172/2165-7939.1000204

\section{Time line for WBC count}

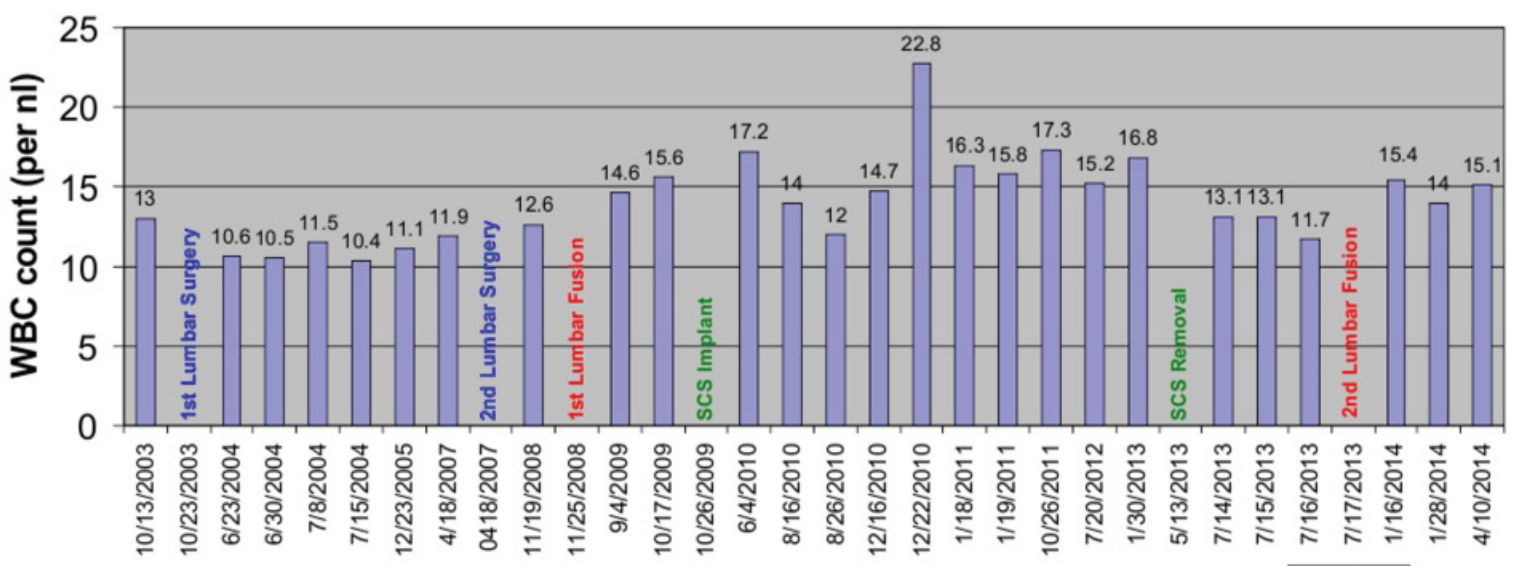

Timeline

\section{口WBC}

Figure 2: Timeline for the patient's WBC fluctuation.

\begin{tabular}{|c|c|c|c|}
\hline Date & $\begin{array}{l}\text { WBC } \\
\text { count }\end{array}$ & Neutrophils \% & Lymphs\% \\
\hline $10 / 13 / 2003$ & 13 & $69 \%$ & $24.60 \%$ \\
\hline $10 / 23 / 2003$ & \multicolumn{3}{|c|}{ Hemilaminotomies and foraminotomies } \\
\hline $6 / 23 / 2004$ & 10.6 & $53.60 \%$ & $38.20 \%$ \\
\hline $6 / 30 / 2004$ & 10.5 & $54.30 \%$ & $34.10 \%$ \\
\hline $7 / 8 / 2004$ & 11.5 & $64.90 \%$ & $28.30 \%$ \\
\hline $7 / 15 / 2004$ & 10.4 & $60.80 \%$ & $30.30 \%$ \\
\hline $12 / 23 / 2005$ & 11.1 & $64.10 \%$ & $27.00 \%$ \\
\hline $4 / 18 / 2007$ & 11.9 & $63.20 \%$ & $30.80 \%$ \\
\hline $0418 / 2007$ & \multicolumn{3}{|c|}{ Laminotomy, medial facetectomy and microdiskectomy } \\
\hline $11 / 19 / 2008$ & 12.6 & $60.20 \%$ & $32.20 \%$ \\
\hline $11 / 25 / 2008$ & \multicolumn{3}{|c|}{$1^{\text {st }}$ Lumbar Fusion } \\
\hline $9 / 4 / 2009$ & 14.6 & $64.40 \%$ & $29.50 \%$ \\
\hline $10 / 17 / 2009$ & 15.6 & $57.90 \%$ & $36.30 \%$ \\
\hline $10 / 26 / 2009$ & \multicolumn{3}{|c|}{ SCS implant } \\
\hline $6 / 4 / 2010$ & 17.2 & $71.90 \%$ & $22 \%$ \\
\hline $8 / 16 / 2010$ & 14 & $66.10 \%$ & $29.10 \%$ \\
\hline $8 / 26 / 2010$ & 12 & $65.20 \%$ & $29.30 \%$ \\
\hline $12 / 16 / 2010$ & 14.7 & $66 \%$ & $26 \%$ \\
\hline $12 / 22 / 2010$ & 22.8 & $57 \%$ & $36 \%$ \\
\hline $1 / 18 / 2011$ & 16.3 & $71.90 \%$ & $23.00 \%$ \\
\hline $1 / 19 / 2011$ & 15.8 & $67 \%$ & $27 \%$ \\
\hline $10 / 26 / 2011$ & 17.3 & $70 \%$ & $23 \%$ \\
\hline $7 / 20 / 2012$ & 15.2 & $72.40 \%$ & $20.70 \%$ \\
\hline $1 / 30 / 2013$ & 16.8 & $67.90 \%$ & $26.20 \%$ \\
\hline $5 / 13 / 2013$ & \multicolumn{3}{|c|}{ SCS Removal } \\
\hline $7 / 14 / 2013$ & 13.1 & $60.50 \%$ & $34.50 \%$ \\
\hline $7 / 15 / 2013$ & 13.1 & $50.50 \%$ & $42.30 \%$ \\
\hline $7 / 16 / 2013$ & 11.7 & $57.90 \%$ & $35.00 \%$ \\
\hline $7 / 17 / 2013$ & \multicolumn{3}{|c|}{ Removal hardware and $2^{\text {nd }}$ lumbar fusion } \\
\hline $1 / 16 / 2014$ & 15.4 & $61 \%$ & $31 \%$ \\
\hline $1 / 28 / 2014$ & 14 & $49.50 \%$ & $26.10 \%$ \\
\hline $4 / 10 / 2014$ & 15.1 & $79 \%$ & $16 \%$ \\
\hline
\end{tabular}

but other causes of elevated WBC count also need to be considered. Currently, the etiology of the persistent leukocytosis is still unknown.

\section{References}

1. Wheeler AH, Stubbart JR, Hicks B (2009) Pathophysiology of Chronic Back Pain, eMedicine, Eds. Medscape

2. Chan CW, Peng $P(2011)$ Failed back surgery syndrome. Pain Med 12: 577-606.

3. Shealy CN, Mortimer JT, Reswick JB (1967) Electrical inhibition of pain by stimulation of the dorsal columns: preliminary clinical report. Anesth Analg 46: 489-491.

4. Boswell MV, Trescot AM, Datta S, Schultz DM, Hanen HC, et al. (2007) Interventional techniques: Evidence based practice guidelines in the management of chronic spinal pain. Pain Physician 10: 7-111

5. Frey ME, Manchikanti L, Benyamin RM, Schultz DM, Smith HS, et al. (2009) Spinal cord stimulation for patients with failed back surgery syndrome: a systematic review. Pain Physician 12: 379-397.

6. Taylor RS (2006) Spinal cord stimulation in complex regional pain syndrome and refractory neuropathic back and leg pain/failed back surgery syndrome: results of a systematic review and meta-analysis. J Pain Symptom Manage 31: S13-19.

7. Turner JA, Loeser JD, Bell KG (1995) Spinal cord stimulation for chronic low back pain: a systematic literature synthesis. Neurosurgery 37: 1088-1095

8. Engle M, Vinh B, Harun N, Koyyalagunta D (2013) Infectious Complications Related to Intrathecal Drug Delivery System and Spinal Cord Stimulator System Implantations at a Comprehensive Cancer Pain Center. Pain Physician16: 251257.

9. Schimmel JJ, Horsting PP, de Kleuver M, Wonders G, van Limbeek J, et al. (2010) Risk factors for deep surgical site infections after spinal fusion. European spine journal: Official publication of the European Spine Society, the European Spinal Deformity Society, and the European Section of the Cervical Spine Research Society 19: 1711-1719.

10. Morais JM, Papadimitrakopoulos F, Burgess DJ (2010) Biomaterials/tissue interactions: possible solutions to overcome foreign body response. AAPS 12: 188-196.

11. Anderson JM, Rodriguez A, Chang DT (2008) Foreign body reaction to biomaterials. Semin Immunol 20: 86-100.

Table 1: The patient's WBC with differentiation over last 10 years. 